\title{
Precise ionospheric electron content monitoring from single- frequency GPS receivers
}

Manuel Hernández-Pajares, 1®,2

Email manuel.hernandez@upc.edu

Manuel Hernández-Pajares is a Full Professor at UPC, Barcelona. He is working on GPS since 1989 (at ICGC at such a time). Since 1994 he has focused on new algorithms for precise ionospheric sounding and GNSS navigation. He has been the chair of the International GNSS Service (IGS) Ionosphere WG (2002-2007), among other projects and responsibilities. He created the new research group, UPC-IonSAT, in Nov. 2013, and presently belongs as well to the IEEC-CTE research group

David Roma-Dollase, 1,3

Email droma@el.ub.edu

David Roma-Dollase is a researcher of the electronics department at the University of Barcelona. He is working as an electronics engineer for the Polarimetric and Helioseismic Imager instrument in the Solar Orbiter mission, developing and testing the correlation camera hardware and firmware. He has also been deeply involved in the validation and software tools for the full image stabilization system. He is also working as assistant professor at the University of Barcelona. At the same time he has also been doing, since 2015, his Ph.D. with the UPC-IonSAT group under the direction of Manuel Hernandez-Pajares, with topics related to real-time ionospheric maps generation and validation. He is also a member of IEEC's ICCUB group

Miquel Garcia-Fernàndez, 4 
Emailmiquel.garcia@rokubun.cat

Miquel Garcia-Fernàndez is an international expert on GNSS precise processing and founder of ROKUBUN S.L. GNSS company in Barcelona, Spain

Raul Orus-Perez, 5

Email Raul.Orus.Perez@esa.int

Raul Orus-Perez is an international expert on GNSS precise processing and staff at the European Space Agency center in ESTEC, Noordwijk, The Netherlands

Alberto García-Rigo, 1,2

Email alberto.garcia.rigo@upc.edu

Alberto García-Rigo is an international expert on GNSS ionospheric determination, space weather and positioning, with a particular focus on real-time/prediction operational systems. He is working as Research Director at UPC, and he has been a co-founder of UPC-IonSAT research group. He is the chairman of IAG's Real-Time Ionosphere Monitoring Working Group, and he has been collaborating with IGS Ionosphere Working Group for the last decade

1 UPC-IonSAT, Universitat Politècnica de

Catalunya, Barcelona, Spain

2 IEEC-CTE-CRAE, Institut d'Estudis Espacials de

Catalunya, Barcelona, Spain

3 Department of Engineering: Electronics, Universitat de Barcelona, Barcelona, Spain

4 Rokubun S.L., Barcelona, Spain 
5

ESA-ESTEC, Noordwijk, The Netherlands

Received: 5 November 2017 / Accepted: 18 July 2018

\section{Abstract}

The number of existing global positioning system (GPS) singlefrequency receivers continues growing. More than 90\% of GPS receivers are implemented as low-cost single-frequency chipsets embedded in smartphones. This provides new opportunities, in particular for ionospheric sounding. In this context, we present the new sidereal days ionospheric graphic (SIg) combination of singlefrequency GNSS measurements. SIg is able to monitor, for each given GNSS transmitter-receiver pair, the vertical total electron content (VTEC) relative to the previous observation with the same or almost the same line-of-sight (LOS) vector. In such arrangements the SIg multipath error mostly cancels, thus increasing the accuracy of the $\triangle$ VTEC significantly. This happens for the GPS constellation after one sidereal day (about $23 \mathrm{~h} 56 \mathrm{~m}$ ) and for Galileo after 10 sidereal days approximately. Moreover, we show that the required calibration of the corresponding carrier phase ambiguity can be accurately performed by means of VTEC global ionospheric maps (GIMs). The results appear almost as accurate as those based on the dual-frequency technique, i.e., about 1 TECU or better, and with much more precision and resolution than the GIM values in the ionospheric region sounded by each given single-frequency receiver. The performance is demonstrated using actual data from 9 permanent GPS receivers during a total solar eclipse on August 21, 2017 over North America, where the corresponding ionospheric footprint is clearly detected in agreement with the total solar eclipse predictions. The advantages of extending SIg to lower carrier frequencies and the feasibility of applying it to other global navigation satellite system (GNSS) systems are also studied. This is shown in terms of a fully consistent VTEC depletion signature of the same eclipse phenomena, obtained with Galileo-only data in North 
America at mid and low latitude. Finally the SIg feasibility, including the cycle slip detection, is shown as well with actual massmarket single frequency GPS receivers at mid and high latitude.

\section{Keywords}

Single-frequency GNSS ionospheric determination

Galileo ionospheric determination

Solar eclipse effects

\section{Introduction}

The interest on ionospheric determination based on single-frequency global navigation satellite system (GNSS) data has recently started (Hein et al. 2016), in parallel to the huge increase in the number of cell phones making single-frequency GPS receivers with increasing performance available to positioning (Gikas and Perakis 2016). Due to the different sign in the ionospheric delay dependency of pseudorange and carrier phase measurements, we can get a biased slant total electron content estimation from their addition. We call this value, divided by two, the ionospheric graphic combination (here in after $I_{\mathrm{g}}$ ). This is the geometry-free counterpart of the standard graphic combination $(G)$, i.e., the ionospheric-free counterpart introduced by Yunk (1992). It is defined, for a given time and GNSS transmitter and receiver, as the mean value of the single-frequency pseudorange and carrier phase measurements. One of the main problems of $G$ and $I_{\mathrm{g}}$ is that they rely on the pseudorange, which are very much affected by multipath and thermal noise, in spite that both are divided by a factor of 2 . We summarize the definition of a new combination of measurements, called the sidereal day difference of Ig, hereinafter called SIg. SIg mostly removes the pseudorange multipath because it is strongly correlated to the repeating transmitter-receiver geometry. In this way, the corresponding sidereal day difference of dual-frequency ionospheric measurements (Hernández-Pajares et al. 1997) is adapted to single-frequency measurements to strongly improve the quality of the electron content difference estimation. A second problem for 
obtaining a precise single-frequency based ionospheric determination is the calibration of the carrier phase ambiguity, in particular for SIg. We will show that the usage of accurate low spatial and temporal resolution global ionospheric maps (GIM) of vertical electron content (VTEC) can provide an excellent calibration of up to and better than 1 TECU. This allows single-frequency permanent GNSS receivers to serve as precise ionospheric sounders with high temporal and spatial resolution in a region around the receiver with a radius of several hundreds of kilometers. This is the case when we consider for calibration the Universitat Politècnica de Catalunya (UPC) VTEC GIMs computed with tomographic and kriging techniques (HernándezPajares et al. 1999, Orus et al. 2005), and identified as "UQRG" by the International GNSS Service (IGS; Dow et al. 2009 and; HernándezPajares et al. 2009, Roma-Dollase et al. 2017).

AQ1

AQ2

\section{Sidereal day filtered ionospheric graphic combination}

Indeed, the ionospheric graphic combination (hereinafter noted as $I_{\mathrm{g}}$, IG, or $I_{\mathrm{G}}$ ) at time $t$ is defined as

$$
I_{\mathrm{G} i}^{j}(t) \equiv \frac{1}{2}\left(P_{1, i}^{j}(t)-\mathrm{L}_{1, i}^{j}(t)\right)
$$

where $P_{1}$ is the pseudorange, $L_{1}$ the carrier phase at a given frequency $f_{1}$, both in length units, $j$ refers to the satellite and $i$ is a permanent receiver.

Because of this definition, the non-frequency dependent terms, such as distance, receiver and satellite clocks, and slant tropospheric delay, cancel. Hence, only the following terms remain:

$I_{\mathrm{G} i}^{j}(t)=I_{1, i}^{j}(t)+\frac{1}{2}\left(D_{1, i}^{j}-B_{1, i}^{j}-\phi_{1, i}^{j}(t)+\mu_{1, i}^{j}(t)-m_{1, i}^{j}(t)+\nu_{1, i}^{j}(t)-\right.$

where $I_{1}$ is the pseudorange ionospheric delay, $D_{1}$ is the $P_{1}$ 
pseudorange instrumental delay, $B_{1}$ stands for the $L_{1}$ ambiguity and contains the transmitter and receiver phase instrumental delays, the unknown integer number of cycles right after the acquisition of the signal; and the carrier phase wind-up is represented by $\phi_{1}$, the multipath and thermal noise $\mu_{1}$ and $v_{1}$ for $P_{1}$ and $m_{1}$ and $n_{1}$ for $L_{1}$, respectively, are all in length units.

We can consider now the operator $\delta(\bullet)=(\cdot)(t)-(\cdot)(t-J \times d)$, where $d$ represents one sidereal day of approximately (23 h $56 \mathrm{~m})$ and coinciding with the repeatability period of the GNSS observational geometry, approximately $J=1$ for GPS and $J=10$ for Galileo.

Furthermore, we can assume that:

- The pseudorange and phase instrumental delays, considered typically constant along 1 day (Hernández-Pajares et al. 2009), approximately cancel: $\delta D_{1, i}^{j} \simeq 0$ and $\delta B_{1, i}^{j} \simeq \lambda_{1} \delta N_{1, i}^{j}$, where $N_{1, i}^{j}$ is the integer number of cycles of the $L_{1}$ measurement of satellite $j$ from receiver $i$. The non-integer part can repeat after 1 day at similar local time, due to similar instrumental operating conditions in general.

- The multipath terms mostly cancel as well due to repeatability of geometry: $\delta \mu_{1, i}^{j} \simeq 0 \simeq \delta m_{1, i}^{j}$.

- The repeated geometry causes the carrier phase wind-up to be almost the same because we are assuming static receivers and $\delta \phi_{1, i}^{j} \simeq 0$.

- The thermal noise of the carrier phase can be considered negligible ( $\simeq 0.002 \mathrm{~m}$ for geodetic receivers) compared to the pseudorange thermal noise ( $\simeq 0.3-3 \mathrm{~m}$ with geodetic receivers and antennas): $\delta \nu_{1, i}^{j}(t)-\delta n_{1, i}^{j}(t) \simeq \delta \nu_{1, i}^{j}(t)$.

- The change of slant ionospheric delay can be expressed in terms of the change of the slant total electron content (STEC), $\delta S_{i}^{j}(t)$, for 
instance, at the $L_{1}$ carrier frequency $f_{1}=154 \times f_{0}$ with $f_{0}=$ $10.23 \times 10^{6} \mathrm{~Hz}$ :

$$
\delta I_{1, i}^{j}(t)=\frac{K}{f_{1}^{2}} \delta S_{i}^{j}(t)
$$

where $K$ is a constant equal to $40.309 \mathrm{~m}^{3} / \mathrm{s}^{2}$ (Hernández-Pajares et al. 2011).

If we express STEC in terms of the mapping function, $M_{i}^{j}(t)$, and the VTEC, $V_{i}^{j}(t)$ :

$$
S_{i}^{j}(t)=M_{i}^{j}(t) \times V_{i}^{j}(t)
$$

We can assume a 2D distribution of the electron density at $450 \mathrm{~km}$ height (Hernández-Pajares et al. 2011), then we write:

$$
\delta I_{1, i}^{j}(t)=\frac{K}{f_{1}^{2}} M_{i}^{j}(t) \times \delta V_{i}^{j}(t)
$$

where we have rewritten the change of slant ionospheric delay using (3) and (4).

Hence, from (2), SIg $\left(\delta I_{\mathrm{G}}\right)$ provides a much simpler and more precise model:

$$
\delta I_{\mathrm{G} i}^{j}(t)=\frac{K}{f_{1}^{2}} M_{i}^{j}(t) \times \delta V_{i}^{j}(t)+\frac{1}{2}\left(-\lambda_{1} \delta N_{1, i}^{j}+\delta \nu_{1, i}^{j}(t)\right)
$$

The only remaining calibration term, $-\lambda_{1} \delta N_{1, i}^{j} / 2$, can be estimated by means of the STEC provided by an accurate VTEC GIM, such as "UQRG”. Hereinafter, $V_{U, i}^{j}(t)$, which is computed in the context of IGS by UPC after applying a combined tomographic and kriging technique, is used in this work. For details see Hernández-Pajares et al. (2017). On the one hand, the GIMs VTEC can be quite accurate, i.e., 
providing a VTEC value at global scale very consistent with independent values from external systems like ionospheric dualfrequency measurements from altimeters, as it is shown in a recent study covering more than one solar cycle (Roma-Dollase et al. 2017). On the other hand, the GIM provides a STEC with significantly less precision than the slant ionospheric information derived from in situ dual-frequency measurements of permanent receivers. This is mainly due to the scarcity of the available permanent GNSS receivers for computing the GIMs in large regions, especially at the southern hemisphere and over the oceans. This also explains that the VTEC GIMs are provided with a low spatial resolution, $5^{\circ} \times 2.5^{\circ}$ in longitude and latitude, and temporal rate, 15 min, compared with the ionospheric information that can be derived from permanent receivers with typical rates of $1-30 \mathrm{~s}$.

Indeed, the $\delta I_{G}$ calibration term, $C=-\frac{1}{2} \lambda_{1} \times \delta N$, can be directly estimated from (6) for each given pair of transmitter $j$, receiver $i$, and continuous arc $k$ of carrier phase $L_{1}$ by realistically assuming its constancy along the continuous-phase arc:

$$
\widehat{C_{U, i, k}^{j}}=<\delta I_{G}-\frac{k}{f_{1}^{2}} M_{i}^{j}(t) \times \delta V_{U, i}^{j}(t)>_{k}
$$

where $<\bullet>$ represents the weighted average along the phasecontinuous arc $k$ of satellite $j$ observed from receiver $i$ and calibrated with a GIM “U”, e.g., UQRG, see for instance Roma-Dollase et al. 2017, with weights $w_{l}$ depending on the elevation angle above the horizon, $E_{i}^{j}(t)$, down-weighting the measurements with low-elevation. In this work we have considered the weights as a Heaviside function, with zero value under $20^{\circ}$ elevation and value 1 above. In this simple way we use observations above $20^{\circ}$ only, avoiding the part with most uncertain of the ionospheric mapping function error:

$$
<\bullet>\equiv \frac{\sum_{l=1}^{N_{k}} \omega_{l}\left[E_{i}^{j}(t)\right] \times(\cdot)_{l}}{\sum_{l=1}^{N_{k}} \omega_{l}\left[E_{i}^{j}(t)\right]},
$$


where $l$ scans all the $N_{k}$ measurements of the phase-continuous arc $k$, with corresponding error $\epsilon_{C_{\mathrm{U}}}$ :

$$
\epsilon_{C_{\mathrm{U}}}=<\frac{1}{2} \delta \nu_{1, i}^{j}(t)>_{k \sim}
$$

derived from (7) and (6).

Moreover, and thanks to the definition of Sig, see (1), the error of its calibration is reduced to half of the pseudorange thermal noise with the multipath mostly canceled. This last point happens because of the repeatability of the line-of-sight geometry, after 1 and 10 sidereal days for GPS and Galileo, respectively (see 2 and 6).

A more simple calibration can be alternatively considered. It is based on the assumption that along the continuous-phase arcs, with typically lasts $2-4 \mathrm{~h}$, the net change of slant electron content relative to the previous reference day is zero:

$$
<\delta S_{i}^{j}(t)>_{k}=<\delta\left(M_{i}^{j}(t) \times V_{i}^{j}(t)\right)>_{k} \simeq 0
$$

This raw approach does not require external information like the VTEC GIMs, but it can be erroneous at the level of few TECUs, showing significant signals above such error level as we will show below. Then the SIg raw calibration, indicated by subindex $R$ in $\widehat{C_{R, i, k}^{j},}$

$$
C_{R, i, k}^{j}=<\delta I_{\mathrm{G}}>_{k}
$$

can be done in a straightforward way, without the need of the external information provided by the GIMs, differently than the previous calibration given in (7).

Once the calibration is performed with any of two strategies, namely based on a GIM $(U)$ or the independent raw one $(R)$, the VTEC daily 
change estimation can be obtained as:

$$
\delta V_{X, i}^{j}(t)=\frac{f_{1}^{2}}{K \times M_{i}^{j}(t)}\left(\delta I_{\mathrm{G}}-\widehat{C_{X, i, k}^{j}}\right)
$$

and its error $\int_{\delta V} \simeq \frac{1}{2} \delta \nu \frac{f_{1}^{2}}{K \times M_{i}^{j}(t)} \in[0.3,0.9]$ TECU for a nominal $P_{1}$ error of $0.3 \mathrm{~m}$ in GPS (Seeber 1993).

\section{SIg validation with permanent geodetic GNSS equipment: solar Eclipse experiment}

The validation of the calibrated SIg is done in a challenging problem: the single-frequency GPS detection of the ionospheric footprint during the recent total solar eclipse over North America happened during August 21, 2017, which occurred at solar minimum, and compare it with the footprint obtained with dual-frequency measurements. Indeed, in top panel of Fig. 1, the location of the 10 receivers analyzed, 9 GPS receivers and one multi-constellation GNSS receiver -SCUB-, close to the total solar eclipse path, are represented. In the bottom panel, the sequential VTEC depletion, $\delta V_{\mathrm{D}}$, is depicted, which has been obtained from the dual-frequency measurements of the GPS receivers with the sidereal day filtering technique described in Hernández-Pajares et al. (1997), hereinafter SI2. The progress of the VTEC depletion is in agreement with the location of the receivers regarding to the advance of the lunar shadow on the ionosphere as predicted by NASA. This can be seen in the top panel and in the above-mentioned reference.

\section{Fig. 1}

Receiver location and VTEC differences. Top panel shows 9 GPS receivers and 1 GNSS receiver (SCUB) considered in the SIg validation, the approximate total (100\%, red line) and partial eclipse boundaries (75\%, yellow lines), and the times predicted by NASA (https://eclipse2017.nasa.gov). The bottom panel shows, for the 9 GPS receivers, the VTEC difference from dual-frequency measurements relative to the previous sidereal day (VTEC_1sd), and calibrated with the UQRG GIM. They are sorted by receiver longitude, from west to 
east, following the moon shadow progress during the total solar in August 21, 2017. To facilitate the comparison, the time series of VTEC differences are shifted in multiples $(k)$ of 5 TECU, from $k=-4$ to +4 from East to West for the 9 stations 


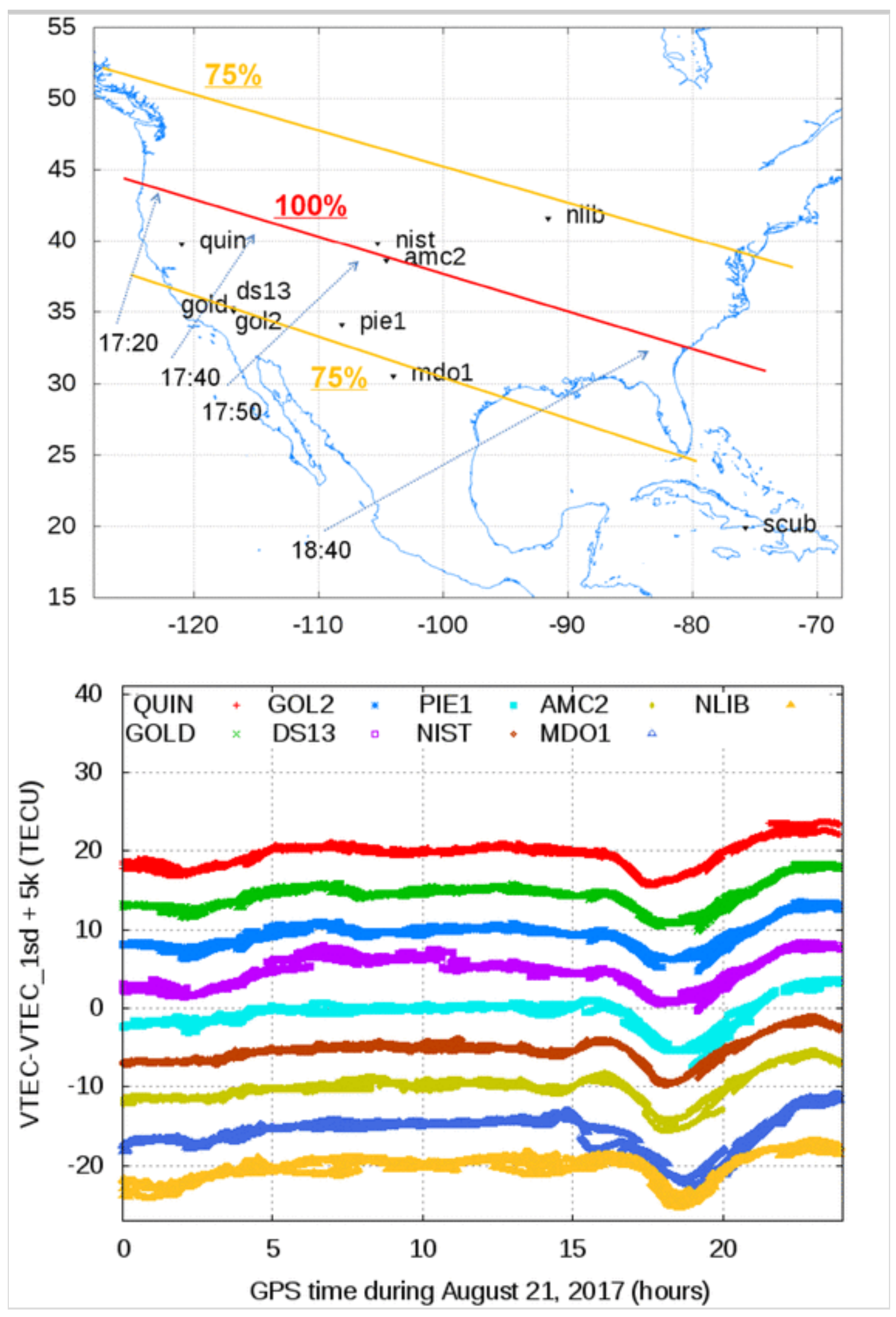

Two representative examples of the detailed validation of single- 
frequency VTEC variation referred to the values of the previous sidereal day, $\delta V_{\mathrm{U}}$ and $\delta V_{\mathrm{R}}$, versus the double-frequency values $\delta V_{\mathrm{D}}$, can be seen in the left panels of Fig. 2. They correspond to the two GPS-only receivers experiencing the smaller and larger VTEC variation during the eclipse, NIST and MDO1 respectively. The very good agreement at sub-TECU level of the VTEC change computed from SIg calibrated with the UQRG GIM VTEC, $\delta V_{\mathrm{U}}$ represented by green points, versus the dual-frequency reference values computed with SI2, $\delta V_{\mathrm{D}}$ corresponding to blue points, is shown. This good performance is also evident in the histograms of the corresponding difference after $15 \mathrm{~h} 00 \mathrm{~m}$, coinciding with the main solar eclipse footprint, see Fig. 2, right panels. The raw calibration, $\delta V_{\mathrm{R}}$ shown as red points, is clearly less accurate, but still captures the most of the progressive VTEC depletion associated to the solar eclipse.

\section{Fig. 2}

Representative examples of the Sig performance during the solar eclipse in August 21, 2017. Left column: Comparison of single-frequency VTEC change after one sidereal day, SIg, determined with UQRG GIM calibration (green points), autonomously calibrated (red points) and from dual-frequency carrier phase measurements, SI2 (blue points), for the analyzed GPS receivers less and most affected by the solar eclipse: NIST (first row) and MDO1 (second row). Right column: Histogram of the difference of the VTEC change with SIg regarding to SI2, both UQRGGIM calibrated, associated to the corresponding left plot. The RMS, bias and standard deviation are also indicated, in TECU, under labels R, B and $\mathrm{S}$ 

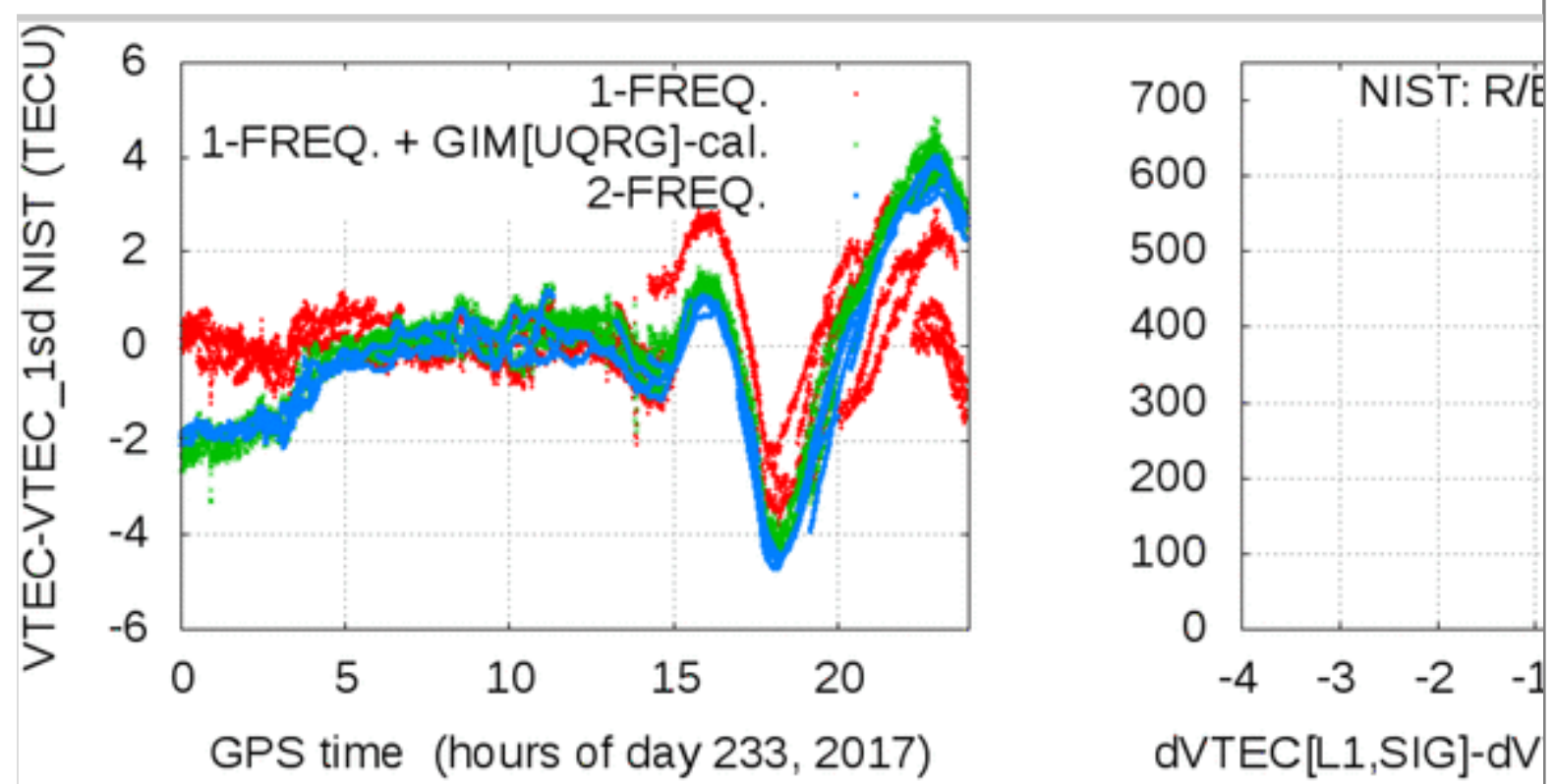

dVTEC[L1,SIG]-dV
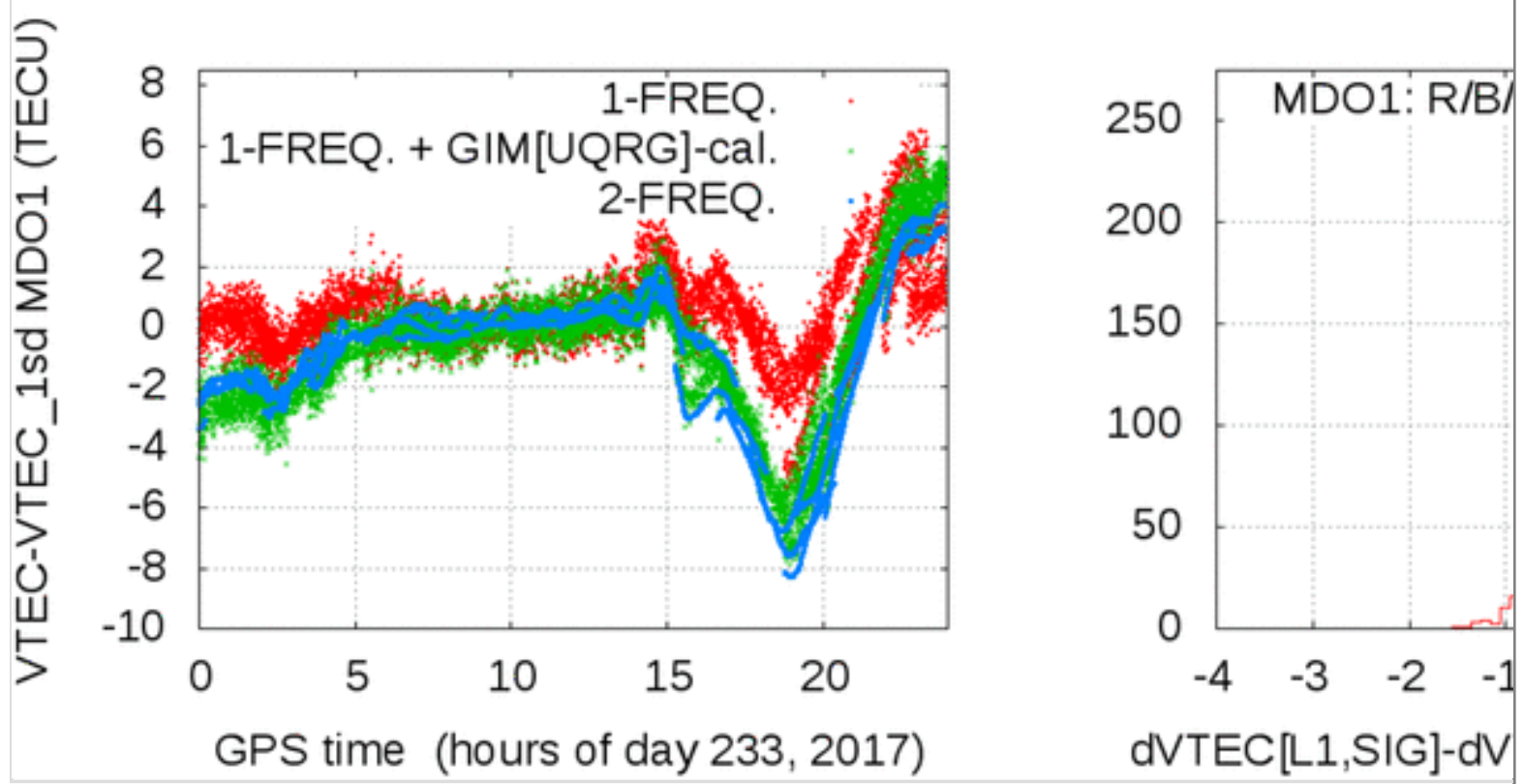

A summary of the SIg performance during the time after 15:00, during the solar eclipse occurrence in the analyzed GPS receivers, can be seen in Table 1. The discrepancy of the single-frequency technique SIg, with respect to the dual-frequency technique SI2, is at 1 TECU or subTECU level, which confirms the very good performance of the proposed GPS single-frequency technique for precise ionospheric monitoring.

AQ3 


\section{Table 1}

Bias, standard deviation, RMS and relative error of the difference of the $s$ determination of change in VTEC

They corr\$\$ \$ 15-24 h). The Peference is the VTEC corresponding to the same satellite-receiver

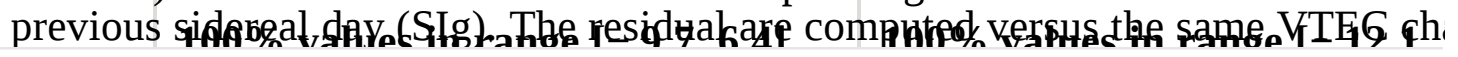

\section{SIg extension to other GNSSs}

We have studied as well the extension of the SIg combination, previously defined for GPS by exploiting the LOS repeatability after one sidereal day for permanent receivers Sig, to other GNSSs like Galileo. Since the main point that might limit such an extension is diminished quality of repeatability of the LOS geometry, and we have focused on the quality. Indeed, it can be seen in the left panel of Fig. 3 that the change in the elevation angle above the horizon for Galileo transmitters tracked from the receiver SCUB, placed at (W76, N20) deg, after approximately 10 sidereal days is quite small, i.e., less than half a degree. A slightly worse repeatability is also seen for GPS after a similar time of approximately 10 sidereal days (see as well top plot of Fig. 3). The validation of the good performance of the strategy can be seen in the bottom plot. In this panel we compare the corresponding SI2-based estimations of VTEC with dual-frequency Galileo data with those of GPS. The comparison has been done for the available multiGNSS receiver SCUB, affected by the same recent solar eclipse of August 2017 over North America. Indeed, it can be seen in the bottom plot that both determinations of $\delta$ VTEC with Galileo-only (green) and GPS-only measurements are fully compatible, showing in particular very clearly the VTEC depletion starting at 17:30, associated with the solar eclipse previously studied. This VTEC decrease at SCUB, taking this case as a reference, the VTEC affecting the receiver 10 days earlier, appears later and deeper compared with previously analyzed GPS stations (Fig. 1, bottom). This is due to the larger longitude and lower latitude of SCUB GNSS receiver (see top plot of Fig. 1). 


\section{Fig. 3}

Consistency of SIg application with Galileo and GPS. Left: Elevation angle change of LOSs corresponding to GPS satellites (red) and Galileo satellites (green), observed from the receiver SCUB for DOY 233, 2017 relative to DOY 223, 2017. Right: VTEC change, in TECUs, during the total eclipse day DOY 233, 2017, referred to approximately 10 sidereal days prior, determined by Galileo $L_{1}-L_{5}$ (green) and GPS $L_{1}-L_{2}$ (red), calibrated with UQRG GIM
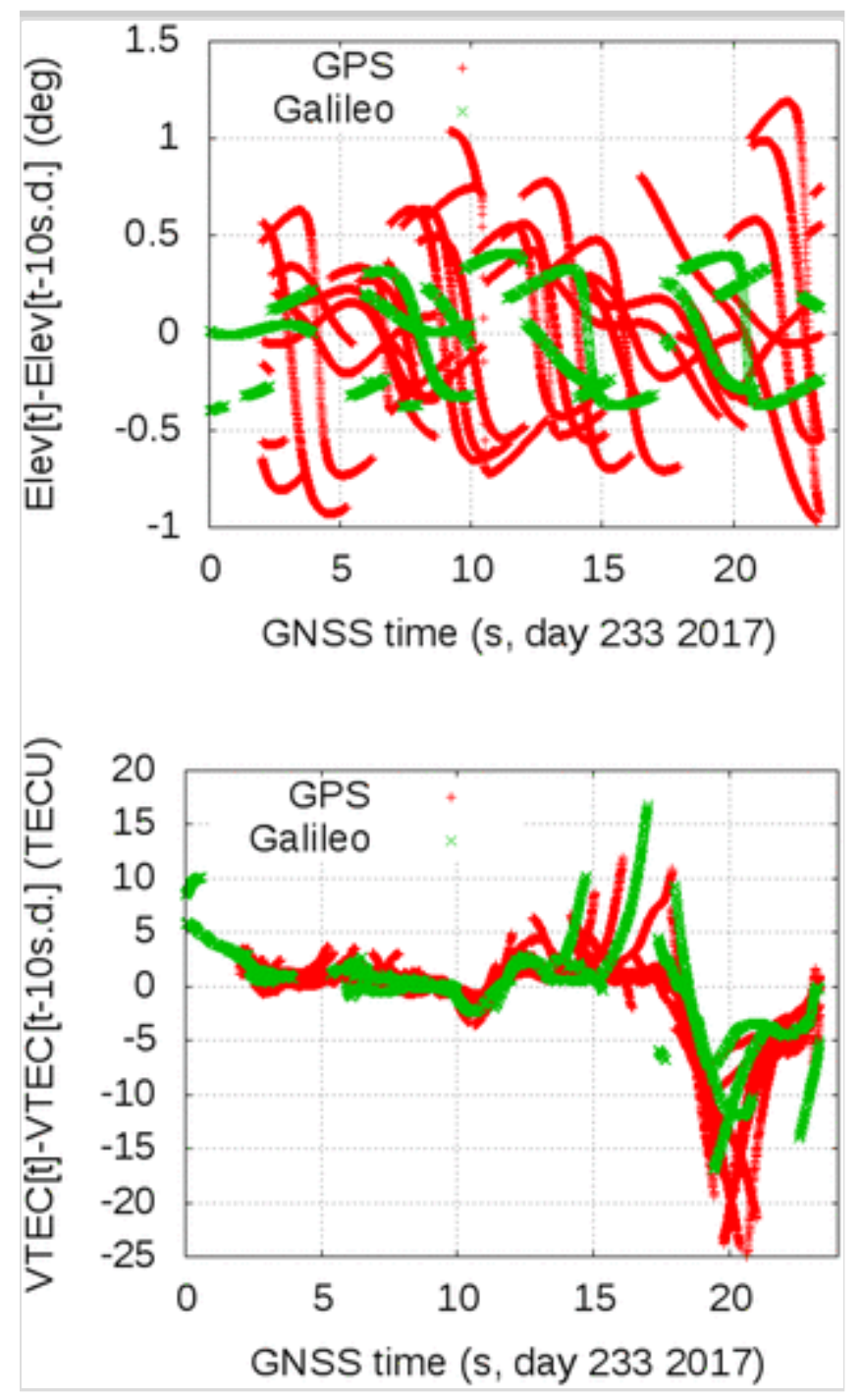

We can conclude this section emphasizing that, even though the ionosphere can be typically more uncorrelated after 10 sidereal days, as compared with 1 elapsed sidereal day, the results shown consistency, 
with a clear observation of the VTEC depletion of the ionosphere for GPS as well as with Galileo (right hand of Fig. 3). The explanation is that the main ionospheric variability periods are the solar-cycle of about 11 years and the seasonal ones of about 6 months. Since both periods are much larger than 10 days, the detrending is not compromised. The only main period, which is still far from the 10 days repeatability of the Galileo LOS electron content, is of about 27 days. This period is typically associated with the solar synodic rotation period and the sun spots, but the amplitude is much smaller (Hernandez-Pajares et al. 2011).

\section{SIg extension to other frequencies}

In the introduction and study of SIg we have selected the first GPS frequency, $f_{1}=1575.42 \mathrm{MHz}$, due to the higher signal-to-noise ratio of $f_{1}$-observations, compared to the $f_{2}$-ones $\left(f_{2}=1227.60 \mathrm{MHz}\right)$. But the frequency of the second carrier is significantly smaller than that of the first carrier, i.e., being more sensitive to the LOS electron content by a factor equal to $\beta=f_{1}^{2} / f_{2}^{2}=(154 / 120)^{2} \simeq 1.65$. Therefore, the ionospheric delay for the same LOS and time for $L_{2}$ is $65 \%$ larger than that for L1.

In order to answer to the question of which effect of SIg2 versus SIg1 can prevail, i.e., the higher noise or the higher ionospheric sensitivity, we have performed the comparison at STEC level, derived independently from $\mathrm{IG}_{1}$ and $\mathrm{IG}_{2}$, respectively, labeled STEC-IG1cal and STEC-IG2cal, all of them calibrated as well with UQRG GIM (see example at top panel of Fig. 4). The performance with different frequencies is shown directly with the calibrated STECs for simplicity, before applying the sidereal day difference $\delta S$. We are comparing also with the direct UQRG-GIM STEC, hereinafter labeled STEC-GIM, and for the sake of completeness, also with the STEC derived from the GIM-calibrated PI, labeled STEC-PIcal. All of them are assessed with respect to the most accurate STEC determination, provided by the GIM calibrated LI, hereinafter STEC-LIcal.

\section{Fig. 4}


Different STEC determinations versus time are shown for receiver SCUB during the day 233, 2017. Top panel: The STEC derived from LI $=L_{1}-L_{2}$ (red), $\mathrm{IG}_{2}$ (light blue), $\mathrm{IG}_{1}$ (magenta) and $\mathrm{PI}=P_{2}-P_{1}$ (dark blue), all of them calibrated with UQRG GIM and the STEC directly given by the UQRG GIM (green). Second row: The corresponding error of calibrated STEC is shown versus time, from calibrated $\mathrm{IG}_{2}$ (left panel) and from calibrated IG $_{1}$ (right panel), taking as reference the STEC from calibrated LI. Third row: Similar to second row, STEC error but taken the STEC directly from GIM (left) and from the GIMcalibrated PI (right) 

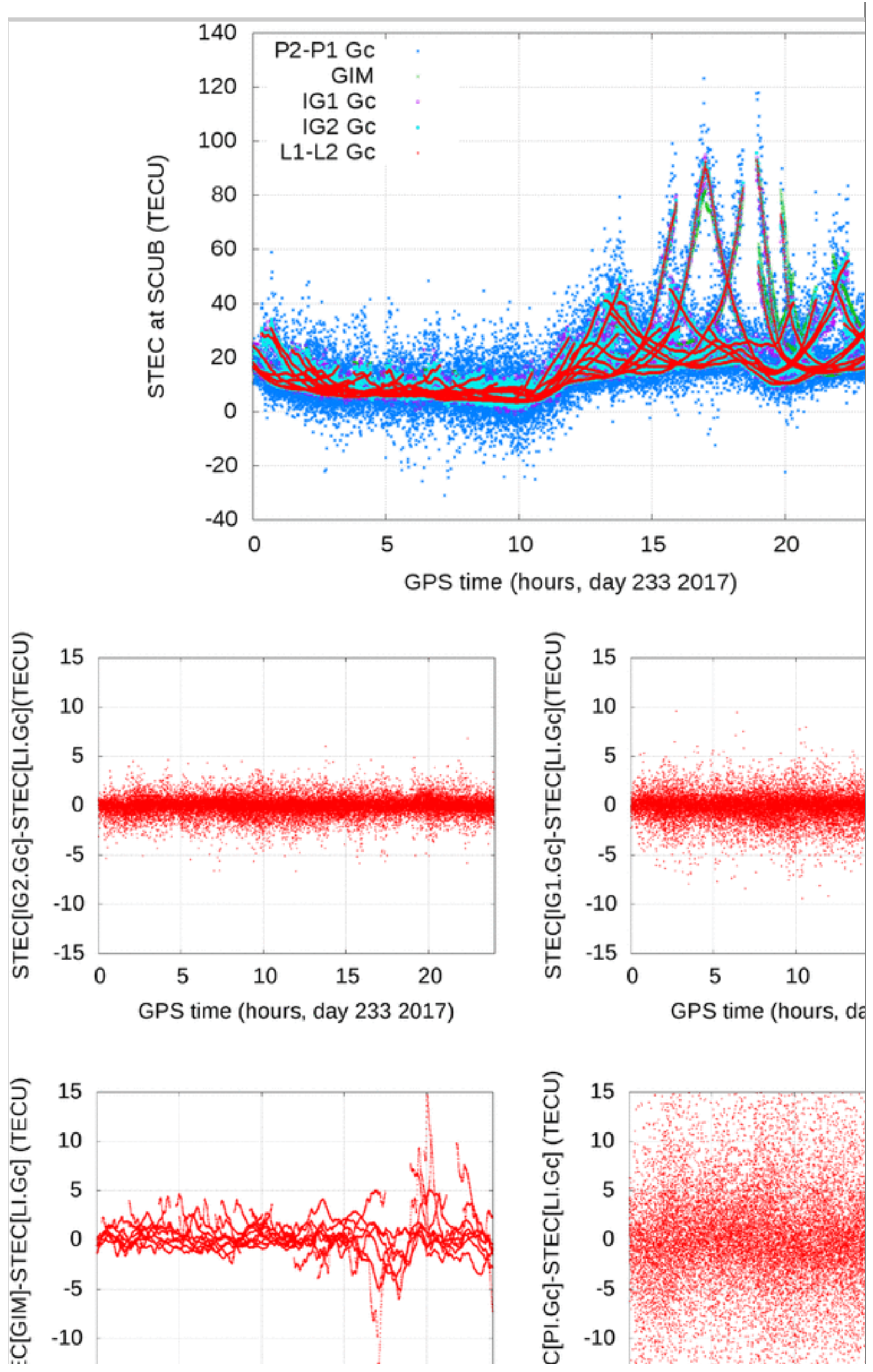
The temporal evolution of the error of the different STEC techniques, regarding the best determination, LI calibrated with UQRG-GIM, is shown for the same receiver SCUB, in panels of second and third row in Fig. 4. It can be seen that STEC-IG2cal performs better than STECIG1cal and STEC-GIM. This last source of STEC given by the GIM is very affected during the solar eclipse due to the relatively poor temporal and spatial resolution of the UQRG GIM: $15 \mathrm{~min}, 5^{\circ}$ and $2.5^{\circ}$ in time, longitude and latitude, respectively. In contrast, the calibrated STEC directly based on observations shows a typical resolution of $30 \mathrm{~s}$ in time, and around $0.25^{\circ}$ in longitude and latitude.

Moreover the statistics of the STEC errors over the 9 GPS and 1 GNSS receivers, for low and high elevation (below and above $45^{\circ}$ ), are shown in Table 2, confirming the best performance of STEC-IG2. For high elevations there is a RMS reduction of $30 \%$ of STEC-IG2 versus STEC-GIM, and 40\% compared with STEC-IG1. Also at lowelevations without multipath mitigation, STEC-IG2 improves about $10 \%$ compared with STEC-GIM and $40 \%$ versus STEC-IG1. This result strongly suggests the potential higher performance of SIg with the new low-frequency GNSS signals (like $f_{5}$ ) which shows a better signal-to-noise ratio, with either slightly lower frequency than $L_{2}$ $\left(f_{5}: f_{2}: f_{1}=115: 120: 154\right)$, combined with multipath correction associated with the sidereal day difference.

AQ5

\section{Table 2}

STEC error RMS, Bias in TEC units (TECU $=10^{16} \mathrm{e} / \mathrm{m}^{2}$ ) and number of involved observations (columns 2-3, 4-5 and 6-7 respectively) corresponding to the 9 GPS and 1 GNSS receivers shown in the top panel of Fig. 1, and taking as reference the STEC values provided by the UQRG-GIM calibrated LI

\begin{tabular}{|l|l|l|l|l|l|l|}
\hline STEC & \multicolumn{2}{|l|}{ RMS } & \multicolumn{2}{l|}{ Bias } & \# Obs. \\
\hline Source & $E \leq 45^{\circ}$ & $E>45^{\circ}$ & $E \leq 45^{\circ}$ & $E>45^{\circ}$ & $E \leq 45^{\circ}$ & $E>45^{\circ}$ \\
\hline
\end{tabular}

The assessed methods, in decreasing order of performance, are calibrated $\mathrm{IG}_{2}$, GIM, calibrated $\mathrm{IG}_{1}$ and calibrated PI 


\begin{tabular}{l|l|l|l|l|l|l|} 
STEC & RMS & \multicolumn{3}{|l}{ Bias } & \# Obs. & \\
\hline Source & $\boldsymbol{E} \leq \mathbf{4 5}^{\circ}$ & $\boldsymbol{E}>\mathbf{4 5}^{\circ}$ & $\boldsymbol{E} \leq \mathbf{4 5}^{\circ}$ & $\boldsymbol{E}>\mathbf{4 5}^{\circ}$ & $\boldsymbol{E} \leq \mathbf{4 5}^{\circ}$ & $\boldsymbol{E}>\mathbf{4 5}^{\circ}$ \\
\hline $\mathrm{IG}_{2} \mathrm{cal}$ & 1.2 & 0.4 & -0.1 & 0.0 & 126,594 & 92,385 \\
\hline GIM & 1.3 & 0.6 & 0.3 & 0.0 & 126,594 & 92,385 \\
\hline $\mathrm{IG}_{1}$ cal & 1.9 & 0.7 & -0.1 & 0.0 & 126,594 & 92,385 \\
\hline PIcal & 8.2 & 3.1 & -0.3 & 0.1 & 126,594 & 92,385
\end{tabular}

The assessed methods, in decreasing order of performance, are calibrated $\mathrm{IG}_{2}$, GIM, calibrated $\mathrm{IG}_{1}$ and calibrated PI

\section{Experiments with mass-market non-permanent single-frequency equipment}

The measurements with a mass-market single-frequency GNSS receiver, taken in two different test cases to assess important aspects, have been analyzed. The receiver belongs to the model Argonaut of Ublox, having an internal patch antenna and commercialized by Rokubun S.L. at a cost of one-order of magnitude lower than the dualfrequency receivers.

\section{Cycle-slip detection: Akureyri experiment (AKUREx)}

The Akureyri experiment (hereinafter AKUREx) has been intended to assess the capability of cycle slip detection with a mass-market singlefrequency receiver, an important aspect to properly process the ionospheric graphic combination. AKUREx was done taking GNSS measurements at $5 \mathrm{~Hz}$ from the Argonaut receiver during almost $12 \mathrm{~h}$ in December 2017, from 12:15 of day 19 to 00:08 of day 20, in an urban canyon test case at high latitude at Akureyri, northern Iceland, with typical high scintillation occurrence. Both characteristics are especially adequate to enhance the occurrence of cycle slips regarding to a normal open-sky mid-latitude situation, for example. In this way, we have been able to study the capability of detecting cycle slips with such a mass-market single-frequency receiver in a challenging test case. 
The approach adopted to detect the cycle slips is to take the Doppler measurement $D_{1}$ as proxy of the carrier phase change $\Delta L_{1}$ among consecutive observations, each $\Delta t=0.2 \mathrm{~s}$, for each given GNSS satellite in view. We can see in Fig. 5, the semi-log histogram plot representing the number of observations for different range of values of the difference $\Delta L_{1}+\lambda_{1} D_{1} \Delta t$, for the more than $1,500,000$ measurements taken. Most of these values are smaller than few centimeters, not showing any cycle slips. Moreover, once a zoom is done and the values of $\Delta L_{1}+\lambda_{1} D_{1} \Delta t$ are expressed in wavelength units, then most of the remaining values appear clustered in multiple of $\lambda_{1}$, instead of having a distribution without local maxima around the integer wavelength values. Such result strongly suggests that not only most of the cycle-slips can be detected, but also fixed, by correcting the corresponding integer number of wavelengths (Fig. 6). AQ7

\section{Fig. 5}

Histogram of the distribution of $\Delta L_{1}+\lambda_{1} D_{1} \Delta t$ values measured in AKUREx, represented for the range of $[-3,+3] \mathrm{m}$ 


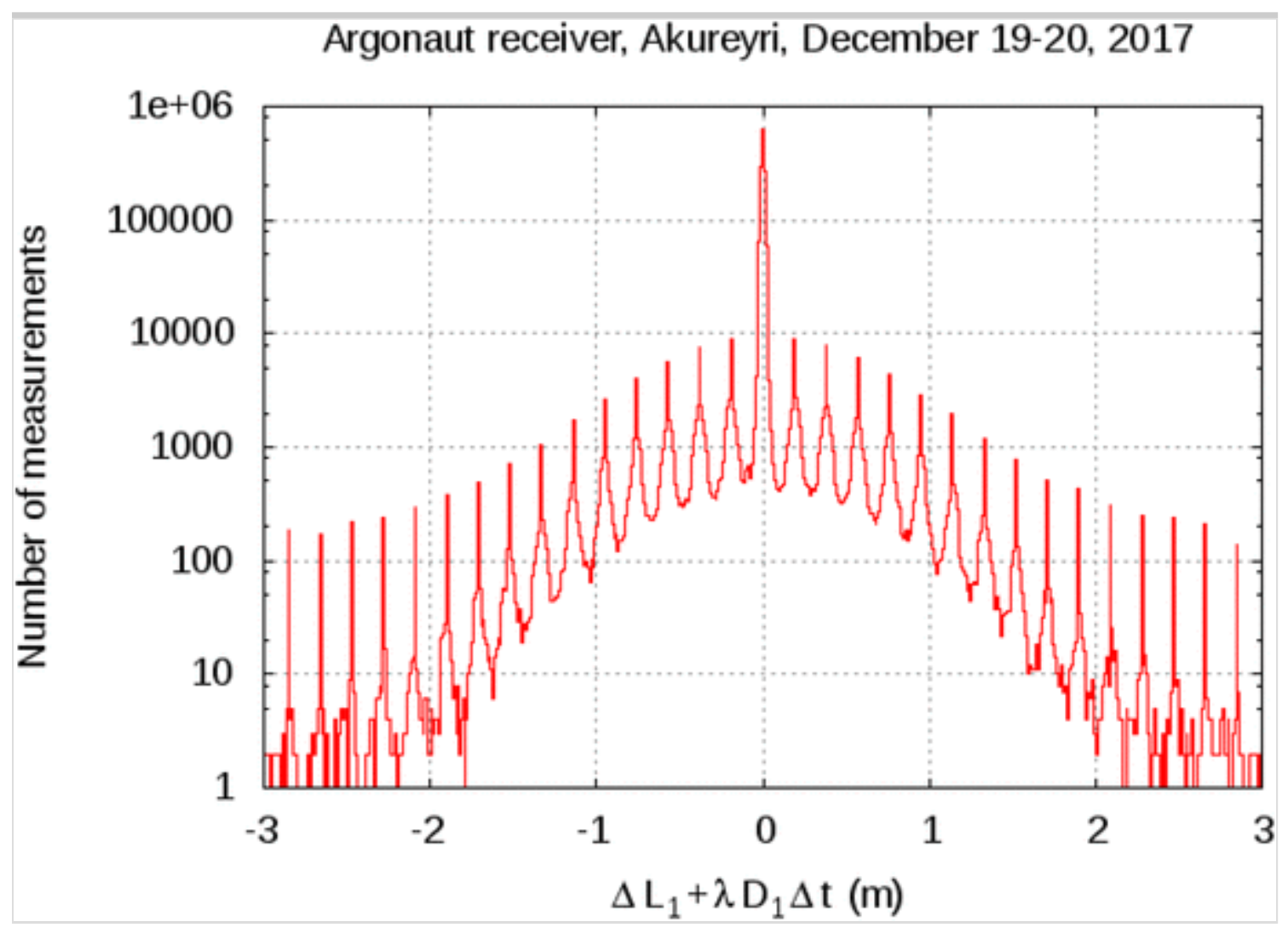

Fig. 6

Histogram of the distribution of $\Delta L_{1}+\lambda_{1} D_{1} \Delta t$ values in wavelength units measured in AKUREx, represented for the range of $[-0.7,+0.7] \mathrm{m}$ 


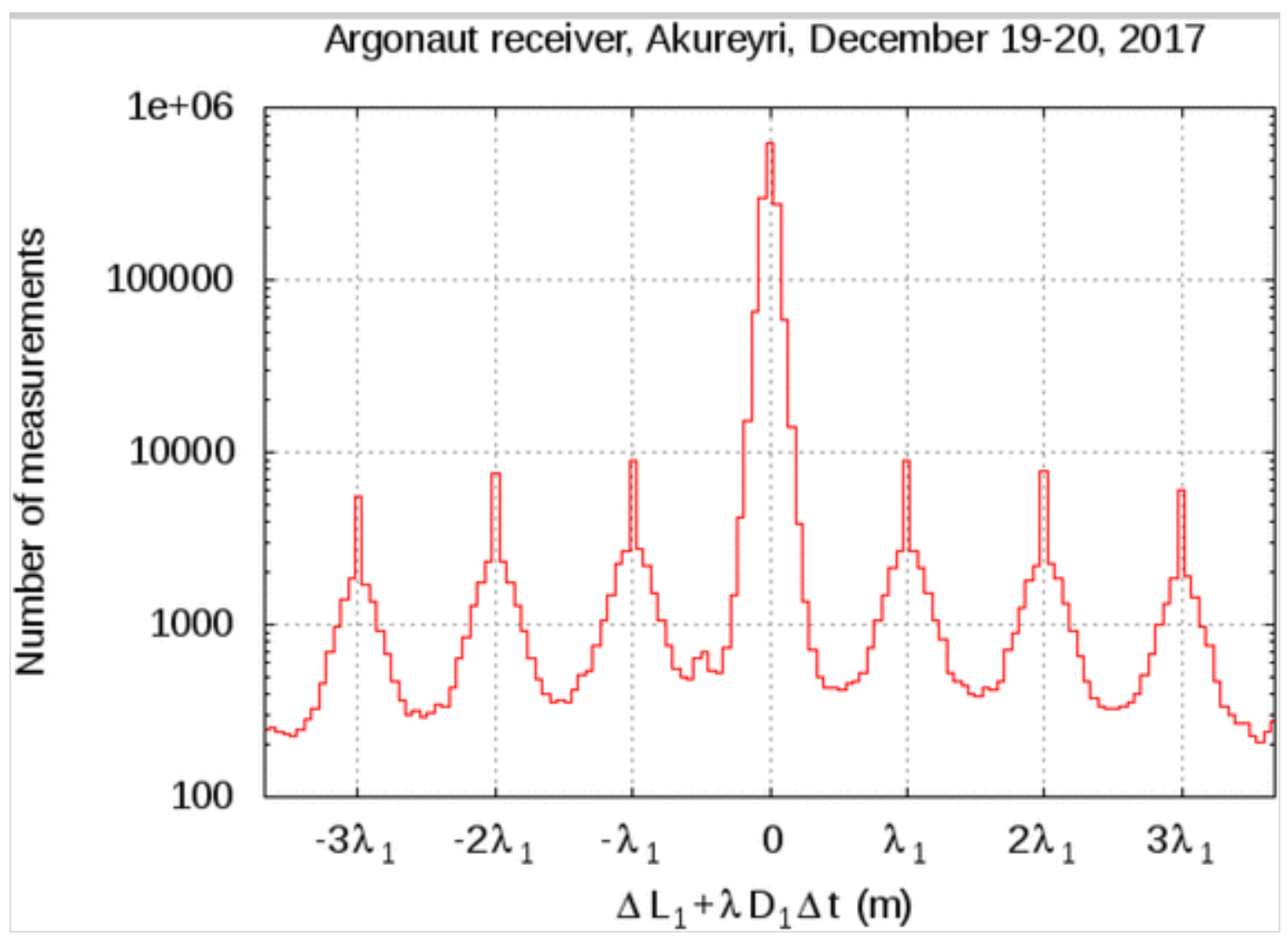

\section{SIg performance: Cornellà experiment (CORNEx)}

To illustrate the SIg performance for current single-frequency massmarket GNSS receivers, the Cornellà experiment (CORNEx) has been performed. We have taken measurements with the Argonaut receiver for several hours during daylight time, revisiting the same point, COR1, approximately after $24 \mathrm{~h}$ on days 24-25, February 2018. The experiment was performed under open-sky and mid-latitude conditions in Cornellà, which is close to Barcelona, Spain. The main objective was to assess the SIg performance with the Argonaut receiver. We will focus on a representative example of one GPS satellite (PRN15) observed for more than 5 consecutive hours. It can be seen in Fig. 7 that the iono-graphic combination, $\mathrm{IG}_{1}$, obtained from the mass-market single-frequency Argonaut receiver shows an error up to 10 TECU (COR1). This error is two to three times the one provided by a geodetic dual-frequency receiver MARE, located a few tens of kilometers away from the Institut Cartogràfic i Geològic de Catalunya, ICGC. The error mainly appears at low elevation, i.e., at the beginning 
and end of the arc. At high elevation the error is similar to that of the geodetic receiver; it is less than 5 TECU when compared with the reference STEC estimation given by the GIM calibrated dualfrequency ionospheric phase combination $L I=L_{1}-L_{2}$. The error is significantly reduced after applying SIg, This is also the case in the Argonaut data due to the predominant repeatability of the multipath after approximately revisiting the same point (Figs. 8, 9). This explains the very high agreement reached with the values based on dualfrequency geodetic grade GNSS receivers, which is better than 1 TECU at high elevation and after smoothing (Figs. 10, 11).

\section{Fig. 7}

Comparison of the STEC obtained from UQRG GIM-calibrated dualfrequency $L I=L_{1}-L_{2}$ (light blue) and from single-frequency $I_{1}=$ $\left(P_{1}-L_{1}\right) / 2$, both from MARE geodetic receiver of the ICGC, which is closely located to our single-frequency receiver at COR1

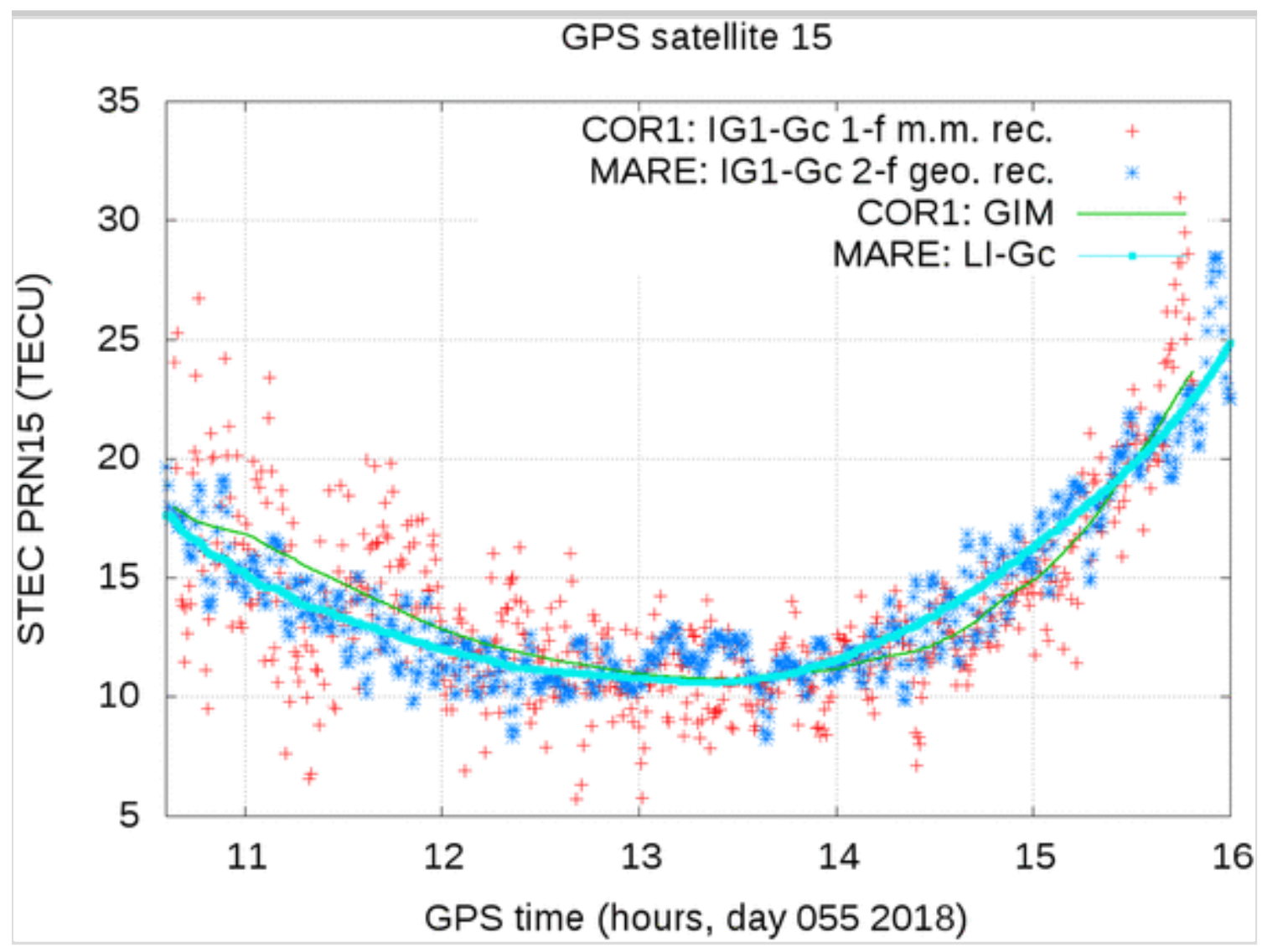




\section{Fig. 8}

Comparison of the STEC obtained from UQRG GIM-calibrated singlefrequency $\mathrm{IG}_{1}=\left(P_{1}-L_{1}\right) / 2$ for the single-frequency receiver at COR1 for February 25, 2018 (day of year 56) and the previous day, shifted 4 min to have both time series aligned in sidereal time

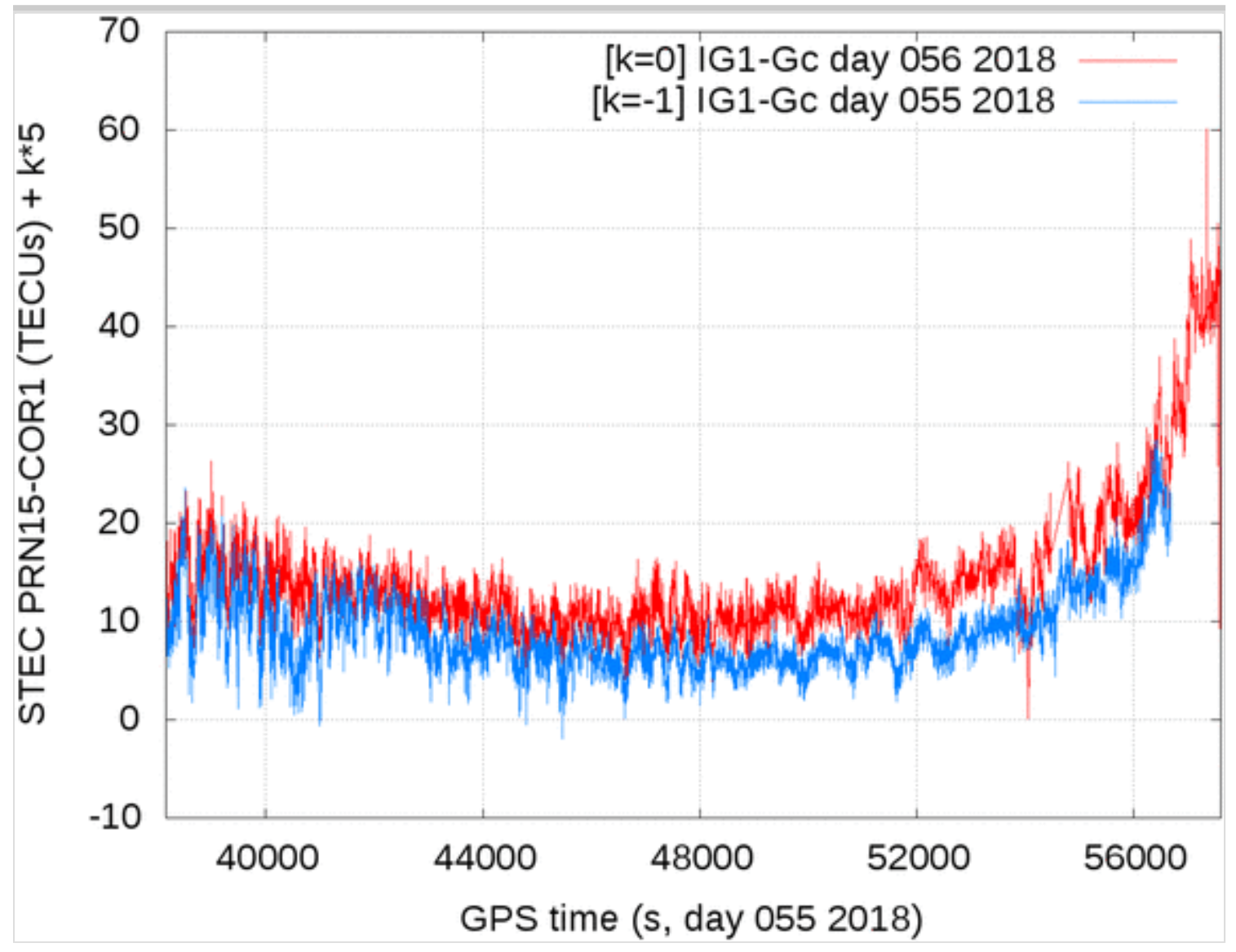

Fig. 9

Zoom of Fig. 8, showing the multipath as a clear repeatable error in the STEC determination after repeating the same geometry, e.g., after one sidereal day approximately 


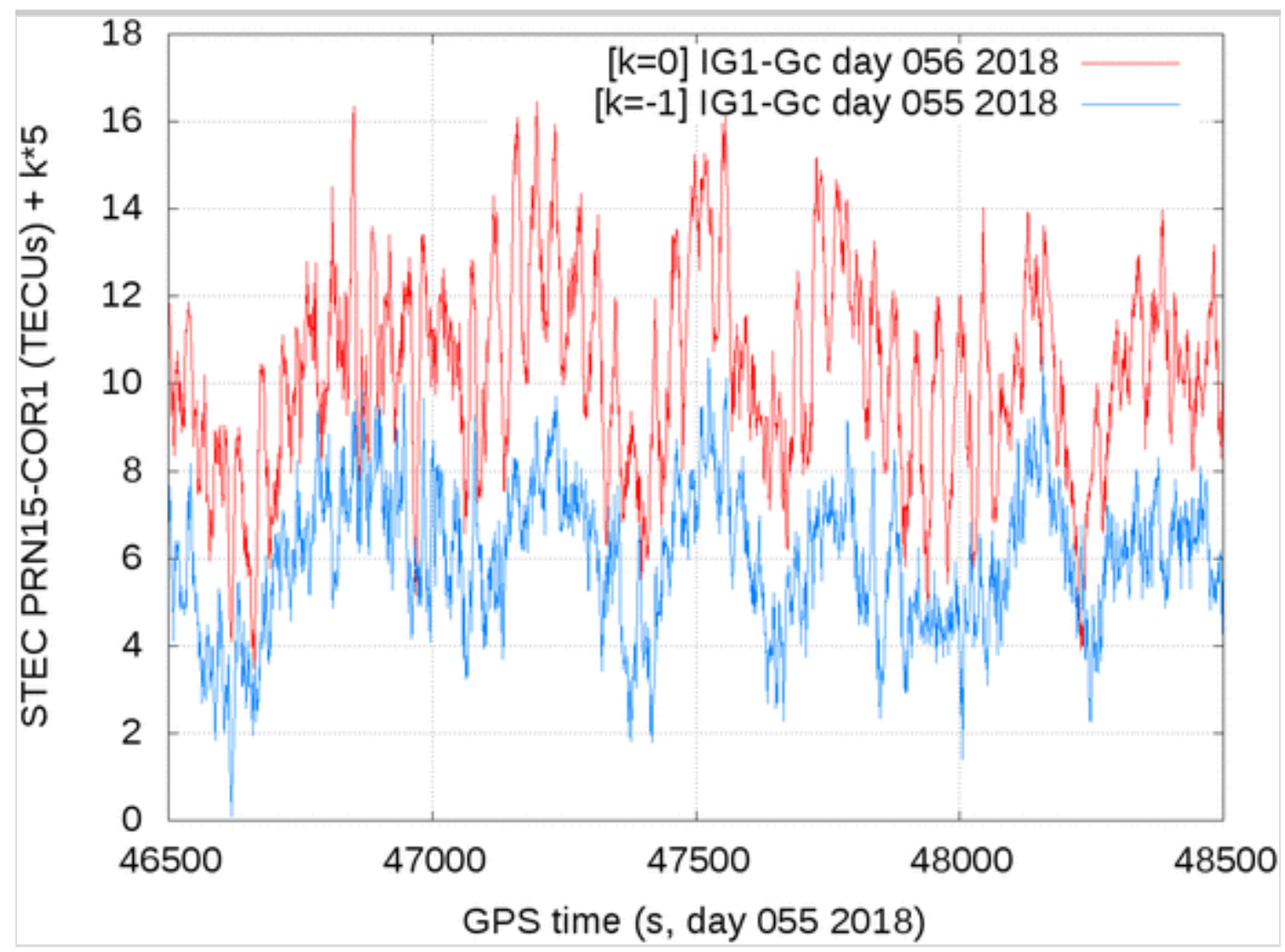

Fig. 10

STEC change obtained from UQRG GIM-calibrated dual-frequency LI $=L_{1}-L_{2}$ (green) and from SIg single-frequency $\mathrm{IG}_{1}=\left(P_{1}-L_{1}\right) / 2$, both for MARE geodetic receiver of the ICGC (blue line), located only few tens of kilometers from our single-frequency receiver at COR1, which $\mathrm{IG}_{1}$ is represented in red, and in magenta after smoothing 


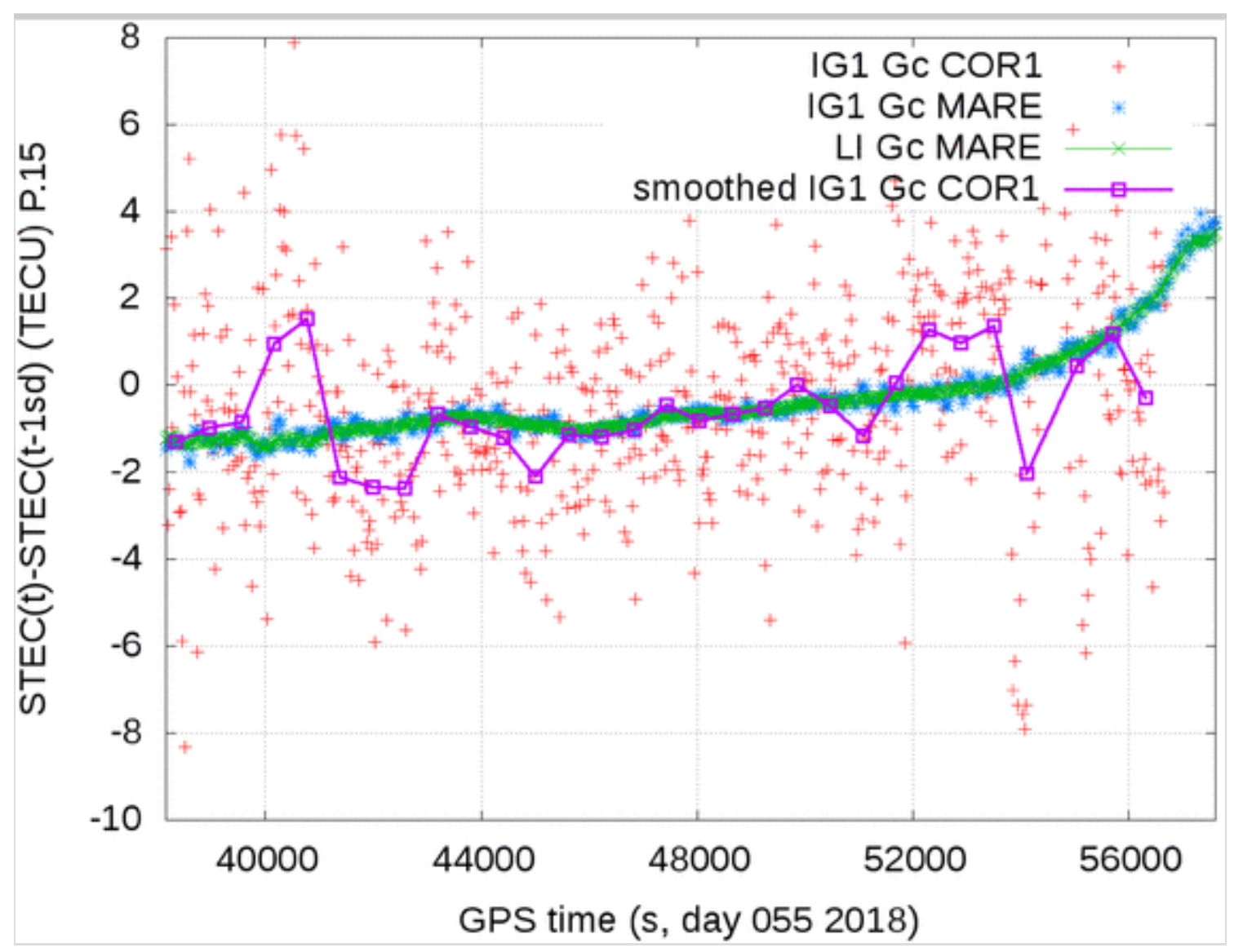

Fig. 11

Zoom of the previous plot showing the sub-TECU agreement between the STEC daily change determined with $\mathrm{IG}_{1}$ from a low cost receiver (magenta points) compared with a geodetic grade receiver and antenna (blue points), versus the reference dual-frequency determination from the same receiver (green points) 


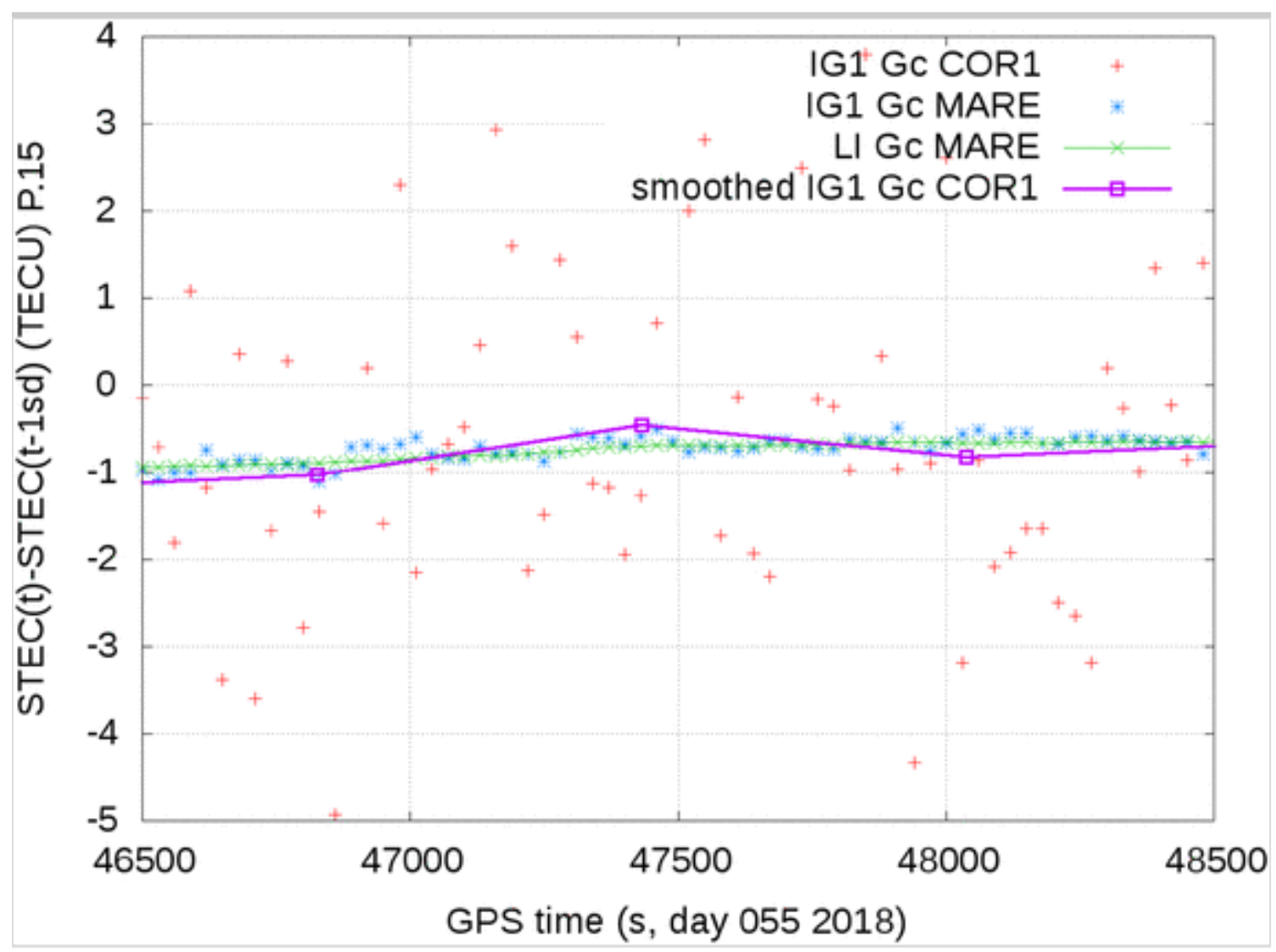

\section{Conclusions}

We presented the new sidereal day ionospheric graphic (SIg) combination, which allows monitoring the VTEC variations with precisions better than 1 TECU from permanently mounted singlefrequency GNSS receivers. This can open future ways of densifying GNSS ionospheric sounding networks with mass-market receivers, complementing the sparsity of dual-frequency receivers in many parts of the world, and able to provide the "absolute" electron content distribution. The SIg performance is shown in the challenging situation of the recent solar eclipse which took place in North America during August 21, 2017. The electron content depletion, due to the advance of the moon's shadow, is clearly seen with the two ways of SIg calibration: based on external VTEC GIMs and based on selfcalibration. Moreover, the feasibility of SIg using measurements of other GNSSs, strongly dependent on LOS geometry repeatability, is shown with Galileo dual-frequency measurements. They provide fully consistent results with GPS and the measured depletion of the eclipse, 
using an approximately 10 sidereal day filtering. Additionally, the advantage of using the SIg associated with new GNSS signals at lower frequencies and good signal-to-noise ratio, like $P_{5}$ and $L_{5}$, is shown in the less favorable case of $L_{2}$ and $P_{2}$ measurements. Finally, the full application of SIg based on mass-market single-frequency receivers is confirmed after analyzing two experiments performed at high and low latitude in Iceland and in Spain.

\section{Acknowledgements}

This work has been done supported by the ESA funded project Atmosfiller-Completing the Atmospheric Sounding System with GNSS and Platform Integrated Sensors (EXPRO + AO8723). The authors acknowledge the IGS data and products. All the data used in this work, the GNSS measurements in both RINEX v2* and v3* formats and VTEC GIMs in IONEX format, are openly available in ftp://cddis.gsfc.nasa.gov/. The first author acknowledges the kind support of Ms. Haixa Lyu providing inputs to the final version of the manuscript.

\section{References}

Dow JM, Neilan R, Rizos C (2009) The international GNSS service in a changing landscape of global navigation satellite systems. J Geodesy 83(3-4):191-198

Gikas V, Perakis H (2016) Rigorous performance evaluation of smartphone GNSS/IMU sensors for ITS applications. Sensors (Basel Switz) 16(8):1240. https://doi.org/10.3390/s16081240

Hein WZ, Goto Y, Kasahara Y (2016) Estimation method of ionospheric TEC distribution using single-frequency measurements of GPS signals. Int J Adv Comput Sci Appl 7(12):1-6

Hernández-Pajares M, Juan JM, Sanz J (1997) High resolution TEC monitoring method using permanent ground GPS receivers.

Geophys Res Lett 24(13):1643-1646 
Hernández-Pajares M, Juan J, Sanz J (1999) New approaches in global ionospheric determination using ground GPS data. J Atmos Solar Terr Phys 61(16):1237-1247

Hernández-Pajares M, Juan J, Sanz J, Orus R, García-Rigo A, Feltens J, Komjathy A, Schaer S, Krankowski A (2009) The IGS VTEC maps: a reliable source of ionospheric information since 1998. J Geodesy 83(3-4):263-275

Hernández-Pajares M, Juan JM, Sanz J, Aragón-Àngel A, GarcíaRigo A, Salazar D, Escudero M (2011) The ionosphere: effects, GPS modeling and the benefits for space geodetic techniques. J Geodesy 85(12):887-907

Hernández-Pajares M, Roma-Dollase D, Krankowski A, GarcíaRigo A, Orús-Pérez R (2017) Methodology and consistency of slant and vertical assessments for ionospheric electron content models. J Geodesy 91(12):1405-1414. https://doi.org/10.1007 /s00190-017-1032-z

Orús R, Hernández-Pajares M, Juan J, Sanz J (2005) Improvement of global ionospheric VTEC maps by using kriging interpolation technique. J Atmos Solar Terr Phys 67(16):1598-1609

Roma-Dollase D et al (2018) Consistency of seven different GNSS global ionospheric mapping techniques during one solar cycle. J Geod 92(6):691-706. https://doi.org/10.1007/s00190-017-1088-9

Seeber G (2003) Satellite geodesy: foundations, methods, and applications. Walter de Gruyter

AQ8 